\title{
Integration of NCD programs in India: Concepts and health system perspective
}

Sitanshu Sekhar Kar, J. S. Thakur ${ }^{1}$

Jawaharlal Institute of Postgraduate Medical Education and Research, Puducherry,

${ }^{1}$ Department of Community

Medicine, School of Public Health, Post Graduate Institute of Medical Education and Research,

Chandigarh, India

Address for the Correspondence:

Dr. J. S. Thakur,

Department of Community Medicine, School of Public Health, Post Graduate Institute of Medical

Education and Research, Chandigarh - 160012 , Punjab, India.

E-mail: jsthakur64@gmail.com

\begin{tabular}{|l|}
\hline Access this article online \\
\hline Website: www.ijmedph.org \\
\hline DOI: $10.4103 / 2230-8598.123386$ \\
\hline Quick response code: \\
\hline
\end{tabular}

Various health programs addressing noncommunicable diseases (NCDs) have been launched in India. Integration of these health programs will avoid duplication of efforts and will address the problem of NCD prevention in a holistic manner. Integration to address the comorbidity due to communicable diseases and NCDs is also required. Various approaches like disease domain, action level, and system level integration should be tried at state level on pilot basis followed by national level integration. Integration of concepts focusing on public health approach and clinical medicine; allopathic system and other Indian System of Medicine; and involvement of private sector in NCD prevention activities will go a long way in combating the already established epidemic in India. Integrated approaches focusing on population and personal clinical services with health system strengthening may be more appropriate strategy for controlling NCDs in India.

Key words: Health system, integration, noncommunicable disease

\section{INTRODUCTION}

"Integration" is the act of combining or adding parts to make a unified whole. The implementation or the process of integrating various sectors in and out of the health sector is critical to achieve overall objective of improving health.

"Integration"; the terminology commonly finds a place in the every policy maker/academician/program manager's address, who is involved in noncommunicable disease (NCD) prevention. As per Collins English dictionary, "Integration" is the act of combining or adding parts to make a unified whole. The implementation or the process of integrating various sectors in and out of the health sector is critical to achieve overall objective of improving health. Health cannot be dealt in isolation. Role of non-health sectors like sanitation and water supply, agriculture, education, urban planning, media, and law also play a vital role in promoting health. Surprisingly very few attempts have been made in the past for integrating their action with health agenda in India. In the context of NCD prevention, there are various levels at which integration can be achieved. This paper describes the types and ways in which integration can be achieved in NCD prevention initiatives.

\section{DISEASE DOMAIN INTEGRATION}

The term NCDs is technically reserved for a group of preventable diseases that are linked by common risk factors. Cardiovascular diseases, chronic obstructive pulmonary disease (COPD) conditions, cancer, and diabetes fall within this category. However, injuries and mental illnesses, which contribute to a substantial burden of diseases, can also be fitted into this framework. However, in India, still mental health and injury prevention is being dealt in isolation and COPD does not find a place in the national program. A new National Tobacco Control Program (NTCP) and National Program for Health Care of Elderly (NPHCE) have also been initiated recently. It will be prudent to initiate an integrated approach for prevention and control of NCDs where cardiovascular disease, cerebrovascular disease, diabetes, COPD, cancer, and mental health find their place. Similar integrated plans have been implemented in neighboring countries like Thailand (1983), Maldives (2001), and recently in Pakistan (2004). $\cdot^{[1-3]}$ 


\section{ACTION LEVEL INTEGRATION}

\section{High-risk approach vs population-based approach}

The paradigm of NCD prevention is multidisciplinary in nature; it calls for a diverse range of actions involving policy development, legislation, regulation, public and professional education, guideline development, media interventions, and research. Monitoring and evaluation need to be woven into this framework, making it necessary to institute a combination of measures and interventions at multiple levels in tandem with effective and rigorous formative research. It should target the at-risk population by adopting the high-risk and population-based approaches set within an enabling policy and regulatory environment. It should encompass two sets of strategies: Those that are common across the entire range of NCDs and others that are specific to each NCD domain. The first strategy maximizes on behavioral change communication, reorientation of health services, and monitoring and surveillance; while the second pertains to legislative and regulatory matters. Population-based strategy will pay rich dividends by reducing the risk factor prevalence across the population and shifting the risk factor distribution to the left side. ${ }^{[4]}$

\section{SYSTEMS LEVEL INTEGRATION}

\section{Vertical vs horizontal approach}

It is time to horizontally integrate the NCD prevention agenda with existing public health, primary healthcare, and social welfare infrastructure. Integration of the prevention of NCDs with the other similar program will bring credibility to the system and avoid duplication of effort. Recently, NCDs have gained importance in India. Various programs namely NTCP, National Mental Health Program (NMHP), NPHCE, and National Injury Prevention Framework are being implemented in phase-wise manner. Currently, MOHFW have integrated cancer control with the national program for prevention and control of diabetes, cardiovascular disease, and stroke. There is a need to integrate these vertical programs and to start a comprehensive integrated NCD prevention program with all the above mentioned components at the district level [Figure 1]. It is encouraging that Ministry of Health and Family Welfare (MOHFW) recently advised states to have single nodal officer for National Program for Prevention and Control of Cancer, Diabetes, CVD, and Stroke (NPCDCS); NPHCE; NTCP; and NMHP at the state and district level.

Integration of NCD related programs is quite possible because of following reasons:

Limited number of risk factors leading to majority of the NCDs Political will is prevailing in the country to address the challenges Evidence shows that integrated approach works

NRHM provides flexibility to include locally prevalent conditions under its umbrella

\section{Structural modification}

There is an immediate need to bring all NCD activities under one administrative unit with financial autonomy. Creation of NCD division or cell at national and state level would be a positive step towards NCD prevention.

\section{Interministerial groups}

Addressing supply and demand side simultaneously is the backbone of any preventive strategy. Till now in India, the focus has been on addressing the demand side, that is, behavior change communication and mass strategy for healthy lifestyle adoption of general population. Very few attempts have made at national level to address the supply side component like making healthy choice easier choice (subsidy on vegetable oils/vegetables/fruits and taxation on tobacco products (which is currently present in Tobacco Act) and alcohol). An interministerial group exists for tobacco control, the scope of which can be enlarged or merged with the broad based group. The ministries involved were agriculture, education, surface transport, rural development, urban planning, and law. This group provides an excellent opportunity to promote intersectoral coordination and many NCD prevention activities can be planned by their help. Health being a state subject, it will be prudent to have such a group at state level under the chairmanship of Chief Minister. It will be the need of the hour to integrate all NCD activities under NRHM framework and under state and district health mission. This will facilitate in implementing state specific policies related to NCD prevention. Health ministry can play the role of convener and perform advocacy regarding NCD burden and implementation of best practices to the group.

\section{INTEGRATION OF CONCEPTS}

\section{Public health approach and curative medicine}

It is widely acknowledged that compared to curative medicine strategies, public health approaches targeting the social determinants of health have a greater impact on health of the people. Disease treatment approach will benefit a few; leaving behind a large number of masses untouched. A public heath approach is population and risk factor oriented rather than symptom or disease oriented as in traditional approaches. Healthcare delivery system should be an optimal mix of population (health promotion) and personal (clinical services). Majority of physicians typically treat signs of illness, but not the risk. But risk reduction may be essential to preventing recurrence of illness. NCDs provides an unique opportunity to target the risk factors as tobacco use, alcohol abuse, unhealthy diet, physical inactivity, and stress accounts for diseases. In NCD prevention, targeting risk factors will improve the overall health status of the community.

\section{Involvement of Indian systems of Medicine (ISM) and social scientist}

Out of the box thinking is required to tackle NCDs in India. Involving qualified and trained people from other sector than allopathy is the need of the hour. Behavioral research, social marketing-guided communication strategy, involvement of Ayurveda, Yoga and Naturopathy, Unani, Siddha, and Homeopathy (AYUSH) practitioners, an active role for local opinion leaders and educational institutions, reorientation of health services to a more preventative orientation, 


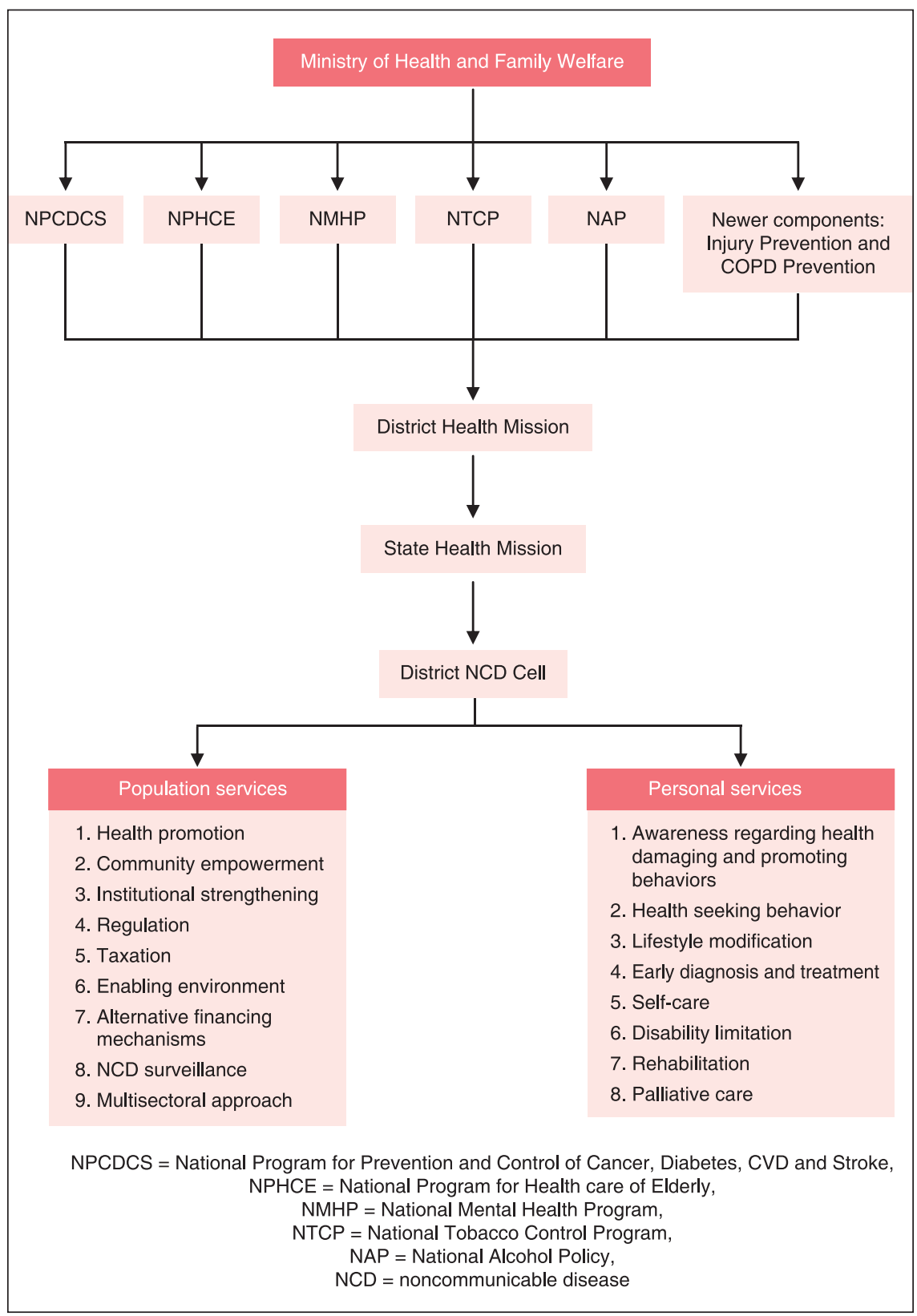

Figure 1: Proposed approach of NCD interventions in India

and a common population surveillance mechanism for all NCDs will go a long way in preventing the rising epidemic.

\section{Public-private partnership}

Private sector caters to around 80 and $50 \%$ of outpatient and inpatient load in India. ${ }^{[5]}$ Involving private sector in public health activities with a scope of interventions that are built on shared responsibility, allowing for agencies to participate according to their own missions, mandates, interests, and resources will increase the reach and scope of NCD prevention. A comprehensive action plan fostering partnership and interface arrangements between the public and private sectors is indispensable. This will reduce the burden of government for getting these programs out to the communities. Proper regulatory mechanisms must be placed to regulate the program implementation in private sector. Such mechanisms have been proved very effective in other programs like RNTCP and Janani Surakha Yojna under NRHM. Furthermore, an action plan for fostering partnerships with international agencies should also be developed.

\section{DISTRICT LEVEL INTEGRATION}

Effective implementation of all NCD prevention activities at the district level is the key to success. Capacity building of program managers to implement such components will be a challenge. A proposed model for integrating all NCD activities is provided below. Mechanism such as District Health Mission exists under NRHM for regular monitoring and implementation of activities of health and non-health sectors. 


\section{The way forward}

Given the potential benefits to be achieved, country-led national responses to NCD should be designed to integrate NCD services within their existing health platforms. In order to make NCD integration a reality, the following steps should be considered:

- Review and reorganization of vertical program structure in Directorate General of Health services (DteGHS)/ MOHFW.

- Careful assessment and documentation of the key lessons learned from current vertical programs and best practices can be replicated.

- Strengthening health system ${ }^{[6]}$ by:

- Providing leadership,

- $\quad$ orienting health system towards prevention (Framework Convention on Tobacco Control (FCTC) implementation, salt intake and, intersectoral collaboration),

- aligning national policies on agriculture, trade, industry, and transport to promote improved diets, increase physical activity, and reduce harmful alcohol use.

- delivering cost-effective and affordable essential drugs and technologies for all priority disorders,

- providing patient-centred care across different levels of the health system, and

- training and orienting healthcare providers to manage and refer chronic disease patients appropriately healthcare delivery, human resources, health financing, essential drugs, and technology and information system

- Development of a country-driven overarching strategy for strengthening of health system.

- Development of evidence-based integration models for various programs implementing the "best buys" after local adaptation. ${ }^{[7]}$

- Development of "how to" kits for the integration of NCD into existing health programs.

- Policy dialogue at the national and international levels to adopt evidence-based integration as a key element of future developmental assistance programs.

- Identifying alternative mechanisms of fund generation. Health cess, money from tobacco/alcohol tax, and health insurance can be some leveraging resources from national governments, donors, private sector, and civil society to support integration implementation.

- Further operational research to define various integration models, what works, what to integrate and what not to integrate, what is cost-effective, and how best to make it work.

\section{REFERENCES}

1. National Action Plan for non-communicable diseases prevention, control and health promotion. Available from: http://www.heartfile.org/napdoc. $\mathrm{htm}$ [Last accessed on May 12 10].

2. Integrated Framework for Action: National Action Plan for noncommunicable diseases prevention, control and health promotion Available from: http://heartfile.org/napIFA.pdf [Last accessed 2010 May 12].

3. Nishtar S. Prevention of non-communicable diseases in Pakistan: An integrated partnership-based model. Health Res Policy Syst 2004;2:7.

4. Rose G. The strategy of Preventive Medicine. Oxford: Oxford University Press; 1992.

5. Misra R, Chatterjee R, Rao S. India Health Report. New Delhi: Oxford University Press; 2003.

6. Beaglehole R, Bonita R, Horton R, Adams C, Alleyne G, Asaria P, et al. Lancet NCD Action Group. Priority actions for the non-communicable disease crisis. Lancet 2011;377:1438-47.

7. From burden to Best buys. Reducing economic impact of NCDs in low and medium income countries. World Economic Forum and the Harvard School of Public Health (2011). Available from: http://www.weforum.org/ Economics Of NCD [Last accessed on 2012 Jan 10].

How to cite this article: Kar SS, Thakur JS. Integration of NCD programs in India: Concepts and health system perspective. Int $\mathrm{J}$ Med Public Health 2013;3:215-8.

Source of Support: Nil, Conflict of Interest: None declared. 